\title{
Carbohydrate Restriction Reduces Lipids and Inflammation and Prevents Atherosclerosis in Guinea Pigs
}

\author{
Moises Torres-Gonzalez ${ }^{1}$, Jeff S. Volek ${ }^{2}$, Jose O. Leite ${ }^{1}$, Heather Fraser ${ }^{3}$, and Maria Luz Fernandez ${ }^{1}$ \\ ${ }^{1}$ Department of Nutritional Sciences, University of Connecticut, Storrs, CT 06269. \\ ${ }^{2}$ Department of Kinesiology, University of Connecticut, Storrs, CT 06269. \\ ${ }^{3}$ Anthera Pharmaceuticals, San Mateo, CA, 944403.
}

\begin{abstract}
Aim: There is limited information on how dietary carbohydrate restriction (CR) or the combination of dietary cholesterol (chol) and CR may affect atherosclerosis development. Guinea pigs were used to evaluate the effects of chol and CR on aortic cholesterol accumulation, mechanical properties of aortas and cytokine production.

Methods: Ten male guinea pigs were fed either low $(\mathrm{L})$ or high $(\mathrm{H})$ chol in combination with $\mathrm{CR}$ or high carbohydrate (control) for $12 \mathrm{wk}$.

Results: Groups fed the high chol (control-H and CR-H) had significantly higher concentrations of cholesterol in aortas and higher activity of serum phospholipase $A_{2}$ than the L groups. CR resulted in significantly lower concentrations of small LDL particles and aortic cytokines and chemokynes than the control groups. Aortas from the control-H and the CR-L were stiffer than those from the control-L and the CR-H groups. This finding could be explained by the reduction in arterial stiffness during the early stages of atherosclerotic.

Conclusion: these results demonstrate that $\mathrm{CR}$ has a major impact on atherogenicity.
\end{abstract}

J Atheroscler Thromb, 2008; 15:235-243.

Key words; Atherosclerosis, Cytokines, Aorta stiffness, Low-carbohydrate diet

\section{Introduction}

Atherosclerosis is the most common pathologic process leading to cardiovascular disease $(\mathrm{CVD})^{1)}$. Although the earliest visible lesion in the development of atherosclerosis is the fatty streak, it is widely recognized that atherosclerosis is a chronic inflammatory disease rather than simply a lipid accumulation problem ${ }^{2)}$. The hydrolysis of phospholipids by the enzyme secretory phospholipase $\mathrm{A}_{2}\left(\mathrm{sPL}_{2}\right)$ is one of the earliest steps in the production of the inflammatory process $^{3,4)}$. This enzyme is responsible for the production of arachidonic acid, which is the precursor of different inflammatory compounds, such as cytokines ${ }^{3,4)}$. These compounds are implicated in the chemoattraction of

Address for correspondence: Maria Luz Fernandez, University of Connecticut Dept. Nutritional Sciences 3624 Horsebarn Road Ext., U-17 Storrs, CT 06269.

E-mail: maria-luz.fernandez@uconn.edu

Received: February 18, 2008

Accepted for publication: May 16, 2008 monocytes, the proliferation of inflammatory cells and the induction of their own secretion, favoring the onset and progression of atherosclerosis ${ }^{2)}$.

In advanced stages of atherosclerosis, the chronic inflammation and the deposition of calcium and atherogenic products, such as lipids and advanced glycated end-products (AGEs), lead to the development of atherosclerotic plaques and increase the stiffness of the arterial wall ${ }^{5-7)}$. This increase in arterial stiffness, associated with the atherosclerotic process, has already been described as a predictor of the morbid-mortality of cardiovascular events (myocardial infarction and stroke $)^{7)}$. Thus, the arterial stiffness is an important parameter to be considered in studies focusing on the effect of interventions to prevent and/or treat atherosclerotic disease.

One of the most common interventions proposed to prevent the development of atherosclerosis is $\mathrm{di}$ etary modification. A number of studies demonstrated that different types of diet can prevent atherosclerosis and reduce the risk for cardiovascular diseases ${ }^{8,9}$. One 
example is the carbohydrate restricted diet (CR), which has been effective in reducing plasma triglycerides (TG), increasing HDL-cholesterol (HDL-C) and decreasing the formation of the more atherogenic, small dense LDL particles ${ }^{8)}$. In relation to inflammation, CR has been shown to reduce plasma levels of different types of cytokines (e.g. tumor necrosis factor-alpha, interleukin- 6 and C-reactive protein $)^{9)}$. In summary, it seems that CR has a hypolipemiant and antiinflammatory effect. Therefore, it is possible that CR can reduce the lipid accumulation in arterial wall and prevent deleterious effects in the aorta's mechanical properties. Our lab group has shown that guinea pigs are an adequate model for the study of atherosclerosis and inflammation ${ }^{10-12)}$. The aim of this study was to evaluate the effect of CR on the factors that contribute to the development atherosclerosis, such as inflammation, lipid accumulation in aorta, sPLA 2 activity, and arterial mechanical properties (stiffness and energy absorption capability) in guinea pigs.

\section{Materials and Methods}

\section{Diets}

Four experimental diets designed to meet the nutritional requirements of guinea pigs were prepared by Research Diets (New Brunswick, NJ). Diets varied in the amount of macronutrients and the concentration of dietary cholesterol. Vitamins, minerals, and fiber were adjusted for the increased caloric content of the CR diets. The composition was as follows: the high carbohydrate-low cholesterol diet (control-L) and the high carbohydrate-high cholesterol diet (Control$\mathrm{H})$ had the following energy contribution: $25.5 \%$ protein: $20.2 \%$ fat and $54.3 \%$ carbohydrate. The carbohydrate restricted-low cholesterol (CR-L) and the carbohydrate restricted-high cholesterol (CR-H) diet had the following energy contribution: $30 \%$ protein, $60 \%$ fat and $10 \%$ carbohydrate. The amount of cholesterol was either low $(0.04 \mathrm{~g} / 100 \mathrm{~g})$ or high $(0.25 \mathrm{~g} / 100 \mathrm{~g})$, equivalent to $300 \mathrm{mg} / \mathrm{d}$ or $1,800 \mathrm{mg} / \mathrm{d}$ in the human situation, respectively ${ }^{13)}$. The high cholesterol was given to develop atherosclerosis in guinea pigs ${ }^{12)}$.

\section{Animals}

Forty male adult guinea pigs weighing between 800-1,000 g were assigned to the four dietary treatments (10 per group). Guinea pigs were housed individually in a metal cage in a light cycle room (light from 07:00-19:00 h) and had free access to water. Food consumption was monitored every other day, and guinea pigs were weighed weekly to ensure stable weight during the 12 wk of treatment. Guinea pigs were deprived of food for $12 \mathrm{~h}$ and sacrificed by heart puncture after isoflurane anesthesia. Blood was collected and plasma separated by centrifugation (200x g) and aortas were harvested and stored at $-80^{\circ} \mathrm{C}$ for further analysis. Animal experiments were conducted in accordance with U.S. Public Health Service/U.S. Department of Agriculture guidelines. Experimental protocols were approved by the IACUC.

\section{Aortic Lipids}

A section of thoracic aorta suspended in formalin was thoroughly cleaned of any excess tissue and fat. Aortic cholesterol concentrations were analyzed as previously reported ${ }^{11)}$. From the cleaned tissue, aortic lipids were extracted from $0.05 \mathrm{~g}$ of aortic arch tissue using $10 \mathrm{~mL}$ of chloroform: methanol (2:1) overnight at RT. The extraction solution was mixed with acidified water to separate into two phases, which were then filtered by gravity filtration. The lower phase was separated and an aliquot of $200 \mu \mathrm{L}$ was evaporated completely and reconstituted with $200 \mu \mathrm{L}$ ethanol for enzymatic determination of free cholesterol ${ }^{12)}$.

\section{Plasma Lipids and Small Dense LDL}

Plasma total cholesterol and LDL-C were measured by enzymatic methods and small dense LDL by nuclear magnetic resonance as previously reported ${ }^{14)}$.

\section{Aortic Cytokines}

The section of ascending aorta, aortic arch, and the upper portion of the descending aorta were processed for cytokine determination. A clean portion of aorta was rinsed with phosphate buffered saline and then cut into small segments and homogenized in 1 $\mathrm{mL}$ of lysis buffer in a rotor - stator (VirTis, Gardiner, NY) on ice for 60-90 sec to ensure complete tissue disruption followed by use of a Potter-Elvehjem homogenizer for additional $60 \mathrm{sec}$. The homogenized tissue was centrifuged at $400 \mathrm{x}$ g for $10 \mathrm{~min}$ at $4{ }^{\circ} \mathrm{C}$ and the supernatant collected and stored at $-80^{\circ} \mathrm{C}$. Twenty-two aortic cytokines were analyzed using a mouse cytokine/chemokine multiplex immunoassay (Lincoplex, Linco Research, St. Charles, MI). Seventeen of the proinflammatory compounds showed good crossreactivity in the aorta of the guinea pigs including 2 chemotactic cytokines (chemokines): monocyte chemotactic protein-1, (MCP-1) and chemokine C-C motif ligand 5 (RANTES) and 13 inflammatory cytokines: tumor necrosis factor-alpha (TNF- $\alpha$ ), interferon-gamma (INF- $\gamma$ ), granulocyte-macrophage colonystimulating factor (GM-CSF), granulocyte colonystimulating factor (G-CSF) and interleukins 1beta, 2, $4,6,7,9,12,13$ and 15 (IL-1 $\beta$, IL-2, IL-4, IL-6, 
IL-7, IL-9, IL-12, IL-13 and IL-15, respectively). Briefly, $25 \mu \mathrm{L}$ of sample were added into the wells of a microplate and mixed with $25 \mu \mathrm{L}$ of mixed beads. The microplate was sealed and covered with aluminum foil and incubated with agitation on a plate shaker overnight $(16-18 \mathrm{~h})$ at $2-8^{\circ} \mathrm{C}$. Next, the fluid was gently removed by vacuum filtration and the microplate was washed two times with buffer, $200 \mu \mathrm{L} /$ well. Subsequently, $25 \mu \mathrm{L}$ of antibody detection cocktail was added to each well, and the plate was sealed and covered with aluminum foil and incubated with vigorous agitation at RT for $60 \mathrm{~min}$. Later, $25 \mu \mathrm{L}$ of streptavidin-phycoerythrin was added to each well and the plate was sealed, covered, and incubated with agitation at RT for 30 minutes. The content of each well was gently removed by vacuum filtration and the plate was washed 3 more times with $200 \mu \mathrm{L} /$ well of wash buffer. Finally, $100 \mu \mathrm{L}$ of sheath fluid was added to all wells and the wells were sealed, covered, and shaken for 5 minutes. The plates were read on a Luminex instrument (Luminex ${ }^{\circledR} 200^{\mathrm{TM}}$ System, Austin, TX.).

\section{Secretory Phospholipase A2 (sPLA2) Activity}

The activity of $\mathrm{SLA}_{2}$ was measured in plasma using Cayman kits (Cayman Chemicals, Ann Arbor, $\mathrm{MI}$ ). Briefly, $10 \mu \mathrm{L}$ of plasma sample were placed with $10 \mu \mathrm{L}$ of DTNB and $5 \mu \mathrm{L}$ of assay buffer in a microplate well. Subsequently $200 \mu \mathrm{L}$ of substrate solution was added and the microplate was carefully shaken. Finally the absorbance at 415 (or 404) nm was read every minute to obtain at least 5 points. Two points on the linear part of the curve were selected and the change in absorbance during that time was determined using the following equation:

$$
\Delta \mathrm{A}_{414}=\left(\mathrm{A}_{414}(\text { Time } 2)-\mathrm{A}_{414}(\text { Time } 1)\right) /(\text { Time } 2-
$$

Time 1).

The following formula to determine sPLA2 activity was used:

$s P L A_{2}$ activity $=\Delta \mathrm{A}_{414} / \mathrm{min} / 10.66 \mathrm{mM}^{-1 *} 0.225 \mathrm{~mL} /$ $0.01 \mathrm{~mL}^{*}$ sample dilution.

${ }_{\mathrm{SPLA}}$ activity was expressed as $\mu \mathrm{mol} / \mathrm{min} / \mathrm{mL}^{15}$.

\section{Aorta Stiffness}

Ring specimens for tensile testing were excised from the proximal portion of descending thoracic aorta and were immersed in saline solution at $4{ }^{\circ} \mathrm{C}$. The cross-sectional area was calculated as follows: cross-sectional area $=2 \times$ thickness $\times$ width. A digital caliper was used to obtain the width of each ring and three-point bend clamps were used in DMA 2980 (Dynamic Mechanic Analyzer) to measure the vessel thickness with a minimum of pre-load force. The artery wall was considered uncompressible for this analysis.

Determination of aorta stiffness was done according to Nosaka et al. ${ }^{16)}$. Briefly, two stainless steel rods were inserted through the lumen of an aorta in a parallel fashion while the vessel was immersed in a saline solution at RT. One rod was attached to a motorized Controller (DMA 2980). This apparatus enabled us to stretch the vessel at a constant tensile force $(0.01 \mathrm{~N})$ while vessel tension was recorded via a force transducer until breakage. Stress (tension per cross-sectional area: $\mathrm{F} / \mathrm{A}$ ) vs. strain (fractional change in vessel width: $((w-w o) / w o))$ curves were generated. Because the obtained curves were non-linear, the elastic modulus was calculated for each point of the graph and modulus was plotted versus stress obtaining a straight line, such as suggested Hayashi et al. ${ }^{17)}$. Thus the slope of each line demonstrates how much the modulus increases per unit of stress, which is an indirect measurement of aorta stiffness ${ }^{17)}$. In addition, the area under the stress-strain curve was calculated by the integral of the curve. This area represents the amount of energy that the aorta can absorb until its breakage. The absorbed energy provides an estimate of the maximum volume of blood that the aorta can accommodate before rupture.

\section{Statistical Analysis}

Two-way ANOVA was used to evaluate carbohydrate and cholesterol effects and the interactions on lipids accumulated in the aorta, sPLA 2 activity, cytokines and aorta stiffness. $P<0.05$ was considered significant.

\section{Results}

\section{Plasma and Aortic Lipids}

The levels of cholesterol accumulated in the aorta were different between the high cholesterol groups (Control-H and CR-H) and the low cholesterol groups (Control-L and CR-L) $(p<0.05)$. The high cholesterol groups had higher concentrations of cholesterol accumulated in the aorta (Control-H: 7.3 \pm 3.11 and CR-H $=6.4 \pm 2.11 \mathrm{mmol} / \mathrm{g}$ ) than the low cholesterol groups (Control-L $=5.4 \pm 3.16$ and $\mathrm{CR}-\mathrm{L}=4.3 \pm 1.06 \mathrm{mmol} / \mathrm{g}$ ). These results clearly indicate that the cholesterol accumulation in the aorta was affected mainly by dietary cholesterol. The same effect was seen for triglycerides accumulated in the aorta. Thus, the high cholesterol diets caused higher plasma triglyceride (Control-H: $34.1 \pm 11.1$ and CR-H: $31.9 \pm 5.41 \mathrm{mg} / \mathrm{g}$ ) accumulation than the low cholesterol diets (Control-L: 20.6 \pm 14.3 and CR-L: $13.6 \pm 7.64 \mathrm{mg} / \mathrm{g})(p<0.001)$.

It is well known that cholesterol accumulation in 


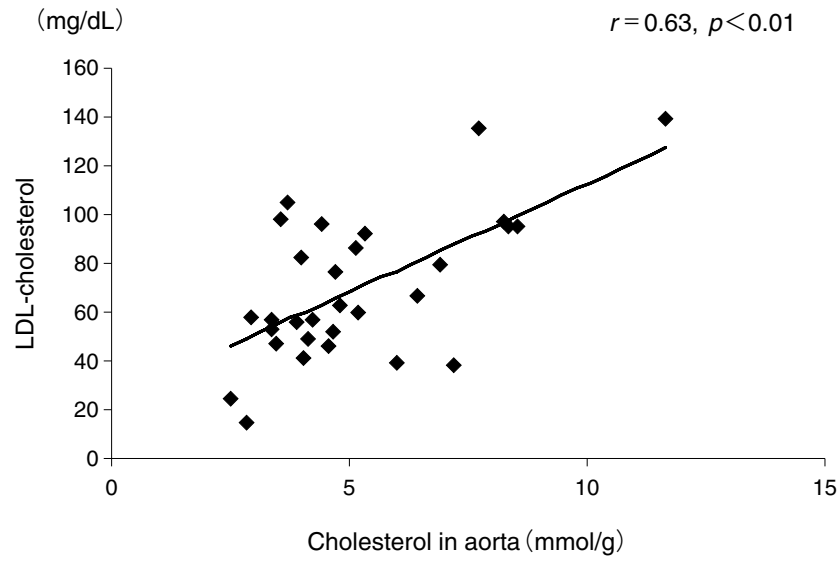

Fig. 1. Correlation between plasma LDL cholesterol and cholesterol accumulated in the aorta for all guinea pigs.

the aorta is closely linked to high levels of plasma LDL-C. In general it is well accepted that the higher the levels of LDL-C, the higher the cholesterol accumulated. Plasma LDL cholesterol concentrations were higher in the $\mathrm{H}$ groups $(2.94 \pm 0.70$ and $2.47 \pm 0.47$ $\mathrm{mmol} / \mathrm{L}$ for Control-H and CR-H, respectively) compared to the $\mathrm{L}$ groups $(1.37 \pm 0.55$ and $1.45 \pm 0.53$ $\mathrm{mmol} / \mathrm{L}$, for Control-L and CR-L respectively $(p<$ $0.01)$. It was also observed that as the LDL-C levels increase in plasma, the cholesterol accumulated in the aorta also increases. They present a positive correlation between these two parameters $(r=0.63, p<0.01-$ Fig. 1). The concentration of small dense LDL subfractions for the 4 groups is shown in Fig. 2. Guinea pigs fed the CR diets had lower concentrations of small dense LDL than those fed the Control diets.

\section{Aortic Cytokines}

Fifteen proinflammatory cytokines were detected in the aorta of guinea pigs. All of these inflammatory markers were found to have a significant carbohydrate effect (Table 1). The high carbohydrate groups had a higher concentration of aortic cytokines than the CR groups, regardless of the level of cholesterol in the diet.

\section{Secretory Phospholipase A2 (sPLA2) Activity}

Guinea pigs fed the high cholesterol diets had higher plasma $\mathrm{SLA}_{2}$ activities compared with guinea pigs fed the low cholesterol diets $(p<0.05)$ (Fig. 3). These results indicated that dietary cholesterol and lipid accumulation in the aorta were the main stimulators of sPLA2 activity.

\section{Aorta Stiffness}

The strain, which is the deformation of the artery,

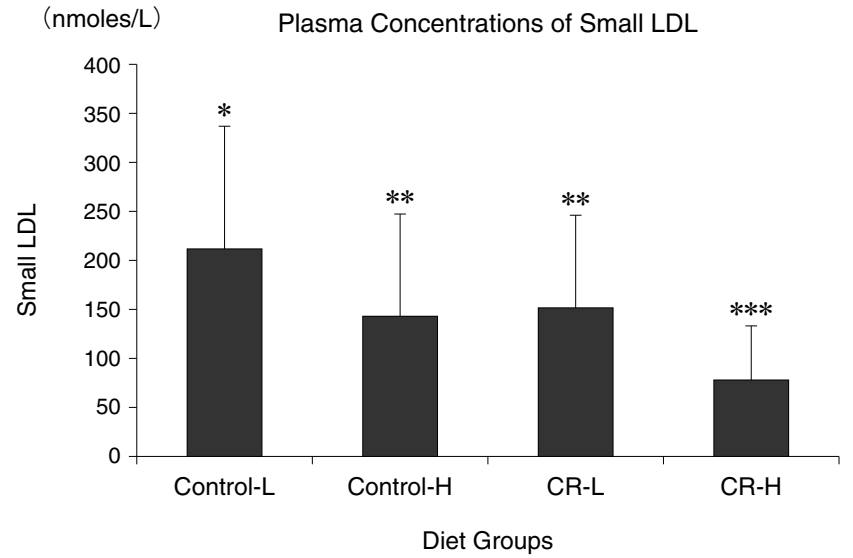

Fig. 2. Concentrations of small dense LDL subfractions fed $54.6 \%$ energy from carbohydrates with low $(0.04 \mathrm{~g} /$ $100 \mathrm{~g})$ (control-L) or high $(0.25 \mathrm{~g} / 100 \mathrm{~g})$ dietary cholesterol (control-H) or 10\% energy from carbohydrates (carbohydrate restricted, CR) with low (CR-L) or high (CR-H) cholesterol.

$* * *$ and ${ }^{* * *}$ indicate significantly different at $p \leq 0.05$.

and the stress, which is the force applied per cross-sectional area were measured to determine the stiffness of the artery. When we plotted stress vs. strain it was observed that the arteries of the CR-L and Control-H groups were stiffer than the arteries of the CR-H and Control-L. This indicates that in order to cause the same degree of deformation in the arteries, more force needs to be applied in the CR-L and Control-H groups (data not shown). However, the stress vs. strain curve is just a general representation of the mechanical behavior of the arteries. A better representation of the properties of the aortas is generating a curve of modulus vs. the stress because it is easier to visualize the stiffness of soft materials such as the arteries (Fig.4). In concordance with the strain-stress curve, the Control-H and CR-L groups had higher elastic modulus/ stress or in other words we may say that were stiffer than the Control-L and CR-H groups $(p<0.05)$. However, aortas from Control-H group demonstrated worse energy absorption capability when compared with the $\mathrm{CR}$ groups. In addition, the groups that received Control diet presented significantly lower capability to absorb energy than those that received CR (Fig. 5). Finally, the groups that received $\mathrm{H}$ presented a trend in reducing the capacity to absorb energy compared with those that received L $(p=0.17)$.

\section{Discussion}

Guinea pigs have been shown to be a good ani- 
Table 1. Concentrations of stimulating factors and cytokines in aortas

\begin{tabular}{lcccccc}
\hline & \multicolumn{2}{c}{ Low Carbohydrate $(\mathrm{pg} / \mathrm{g})$} & & \multicolumn{2}{c}{ High Carbohydrate $(\mathrm{pg} / \mathrm{g})$} & \multirow{2}{*}{ Carbohydrate Effect } \\
\cline { 2 - 3 } Parameter & Low Chol & High Chol & & Low Chol & High Chol & \\
\cline { 2 - 3 } IL-1 $\beta$ & $92.1 \pm 19.3$ & $116.1 \pm 7.0$ & & $194.6 \pm 52.6$ & $261.6 \pm 24.3$ & \\
IL-2 & $64.5 \pm 16.7$ & $63.4 \pm 5.9$ & & $107.4 \pm 29.1$ & $122.5 \pm 6.5$ & $0.007^{* *}$ \\
IL-4 & $116.2 \pm 25.8$ & $137.4 \pm 7.1$ & & $253.6 \pm 69.0$ & $290.8 \pm 29.9$ & $0.048^{*}$ \\
IL-7 & $100.3 \pm 21.9$ & $133.9 \pm 10.1$ & & $244.9 \pm 67.8$ & $293.5 \pm 34.2$ & $0.014^{*}$ \\
IL-6 & $103.1 \pm 22.0$ & $126.8 \pm 7.7$ & & $195.6 \pm 47.6$ & $269.4 \pm 34.9$ & $0.006^{* *}$ \\
IL-9 & $100.6 \pm 22.3$ & $110.3 \pm 7.8$ & & $193.3 \pm 52.0$ & $257.7 \pm 20.2$ & $0.006^{* *}$ \\
IL-12 & $109.5 \pm 22.5$ & $147.8 \pm 9.6$ & & $253.3 \pm 68.7$ & $307.5 \pm 27.4$ & $0.008^{* *}$ \\
IL-13 & $108.1 \pm 25.4$ & $105.1 \pm 11.1$ & & $213.1 \pm 61.2$ & $268.1 \pm 18.1$ & $0.009^{* *}$ \\
IL-15 & $99.9 \pm 20.0$ & $122.5 \pm 7.4$ & & $225.4 \pm 63.2$ & $290.3 \pm 31.2$ & $0.011^{*}$ \\
IFN- $\gamma$ & $85.5 \pm 19.1$ & $86.6 \pm 5.6$ & & $149.3 \pm 40.1$ & $196.0 \pm 14.8$ & $0.007^{* *}$ \\
TNF- $\alpha$ & $92.1 \pm 20.4$ & $116.2 \pm 9.0$ & & $207.4 \pm 55.6$ & $249.7 \pm 28.4$ & $0.014^{*}$ \\
GM-CSF & $93.6 \pm 20.7$ & $117.1 \pm 10.5$ & & $212.7 \pm 56.4$ & $249.5 \pm 30.9$ & $0.010^{* *}$ \\
G-CSF & $119.8 \pm 26.4$ & $152.1 \pm 10.9$ & & $253.4 \pm 70.6$ & $320.9 \pm 31.7$ & $0.011^{*}$ \\
MCP-1 & $73.3 \pm 17.8$ & $90.2 \pm 9.5$ & & $153.8 \pm 41.1$ & $190.4 \pm 18.6$ & $0.014^{*}$ \\
RANTES & $101.7 \pm 22.2$ & $104.0 \pm 10.7$ & & $186.5 \pm 48.1$ & $261.0 \pm 23.5$ & $0.011^{*}$ \\
\hline
\end{tabular}

Values are presented as mean \pm SEM for $n=10$ guinea pigs per group. ${ }^{*}$ for $p \leq 0.05$ and ${ }^{* *}$ for $p \leq 0.01$.

Concentration of SPLA2

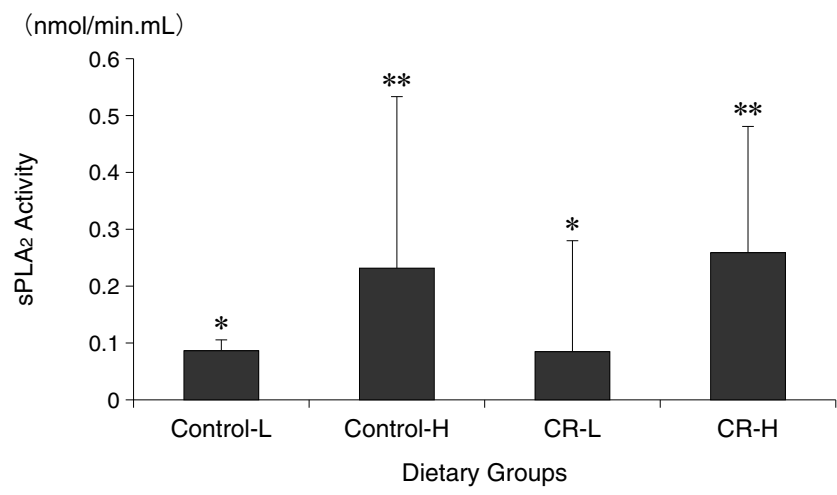

Fig. 3. Concentrations of $\mathrm{sPA}_{2}$ of guinea pigs fed $54.6 \%$ energy from carbohydrates with low $(0.04 \mathrm{~g} / 100 \mathrm{~g})$ (Control-L) or high $(0.25 \mathrm{~g} / 100 \mathrm{~g})$ dietary cholesterol (Control-H) or 10\% energy from carbohydrates (carbohydrate restricted, CR) with low (CR-L) or high (CR-H) cholesterol.

$* * *$ and $* * *$ indicate significantly different at $p \leq 0.05$.

mal model for the study of atherosclerosis and inflammation ${ }^{10)}$. Because human studies over the last decade have shown that CR diets improve plasma lipid profiles and reduce atherogenic lipoproteins, we used guinea pigs in the current study to evaluate the effects of CR on the different aspects of the onset and progression of atherosclerosis, such as: lipid accumulation in the aorta, sPLA 2 activity, inflammation, and arterial stiffness ${ }^{18)}$.

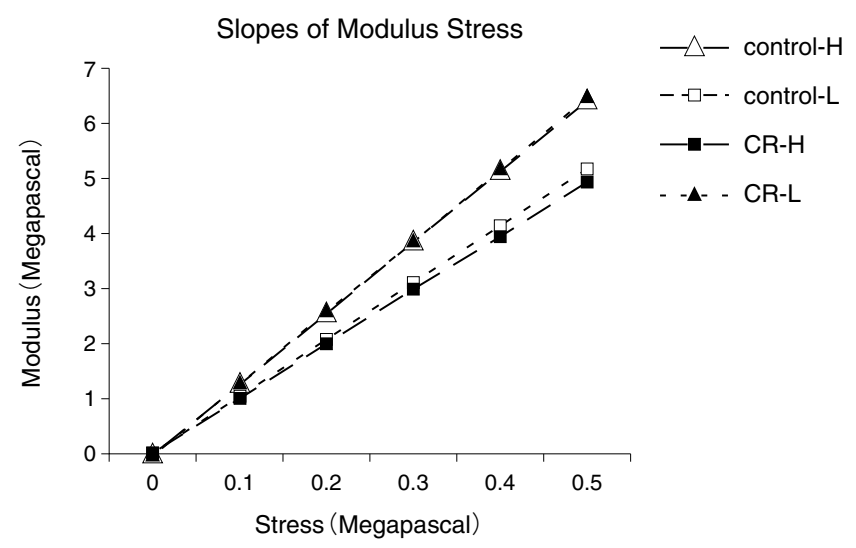

Fig. 4. Curves of modulus/stress of the aortas of guinea pigs fed 54.6\% energy from carbohydrates with low $(0.04$ $\mathrm{g} / 100 \mathrm{~g})$ (Control-L) or high $(0.25 \mathrm{~g} / 100 \mathrm{~g})$ dietary cholesterol (Control-H) or 10\% energy from carbohydrates (carbohydrate restricted, CR) with low (CR-L) or high (CR-H) cholesterol.

* for $p \leq 0.05$.

\section{Cholesterol Effects}

One of the major causes of atherosclerosis is lipid accumulation in the aorta. It is accepted that hypercholesterolemia is related to the onset and progression of atherosclerosis and that plasma LDL-C concentrations is the major contributor ${ }^{19)}$. In agreement with these observations, in the current study we observed that high cholesterol diets not only caused elevated LDL-C levels but also increased the cholesterol accu- 


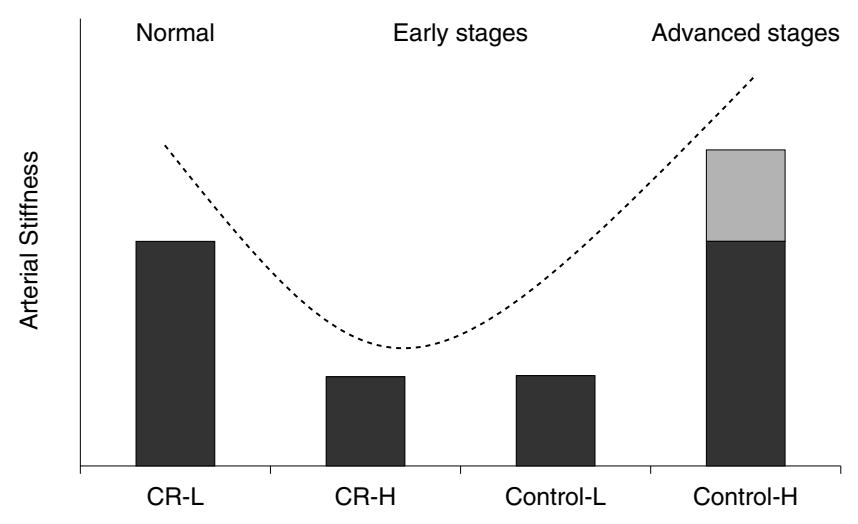

Fig. 5. Area under the stress-strain curves. of guinea pigs fed $54.6 \%$ energy from carbohydrates with low $(0.04 \mathrm{~g} /$ $100 \mathrm{~g})$ (Control-L) or high $(0.25 \mathrm{~g} / 100 \mathrm{~g})$ dietary cholesterol (Control-H) or 10\% energy from carbohydrates (carbohydrate restricted, CR) with low (CR-L) or high (CR-H) cholesterol.

$*, * *$ and $* * *$ indicate significantly different at $p \leq 0.05$.

mulated in the aorta. The latter variables were significantly correlated.

High cholesterol diets also affected TG accumulation in the aorta. Surprisingly, a high carbohydrate intake did not show such effect. Usually, carbohydrate effect on TG accumulation result from a higher caloric intake associated with high carbohydrate diets. In the present study, however, the caloric intake was not different among groups, which may explain the lack of carbohydrate effect. Thus, the explanation for the fact that the highest aortic TG accumulation occurred in $\mathrm{H}$ groups is based in the lipoprotein profile. LDL, which is a TG rich lipoprotein, is the most important molecule that accumulates in arteries. Especially after the action of sPLA2, LDL particles become smaller and denser, presenting higher affinity to the extracellular matrix (ECM). This fact favors the lipoprotein trapping in arterial wall and the formation of foam cells $^{20)}$. Finally, since LDL particles carry both CHOL and TG, the increase in CHOL and TG in aorta is explained by the same factor: the higher concentrations of LDL in plasma of animals that received $\mathrm{H}$.

It is known that $s \mathrm{PLA}_{2}$ is mainly produced and secreted by the proinflammatory cells macrophages/ monocytes, mast cells, and $T$ lymphocytes, which play a critical role in the onset and advance of atherosclerosis $^{21)}$. sPLA2 participates in the hydrolysis of membrane phospholipid-derived inflammatory mediators such as arachidonic acid, which is involved in the synthesis of platelet-activating factor and prostaglandins, important components at the beginning of the inflammatory cascade ${ }^{19,22)}$. Moreover, sPLA2 hydrolyzes oxi- dized phospholipids, generating lysophosphatidycholine and free oxidized fatty acids ${ }^{23)}$. Thus, this enzyme is responsible for most of the increased lysophosphatidycholine content of oxidized LDL particles. This is the first study in guinea pigs showing increased sPLA 2 activity by high cholesterol diets.

An interesting observation is that the groups with higher sPLA2 activities also had higher cholesterol accumulated in the aorta, suggesting that sPLA 2 could have been produced by those inflammatory cells located in the area of lipid accumulation.

Although the sPLA2 activity was greater in $\mathrm{H}$ groups, the inflammation is higher in Control groups. Hence, while it seems that the inflammation in $\mathrm{H}$ groups was modulated by the action of sPLA2, Control seems to promote inflammation through other pathways, such as AGEs stimulation ${ }^{24-26)}$. This is consistent with the fact that this enzyme hydrolyzes lipids. Thus, the greater the lipid accumulation, the greater the $s \mathrm{PLA}_{2}$ activity. Future studies should be done to uncover the pathways for increased inflammation promoted by high carbohydrate intake.

\section{Carbohydrate Restriction Effects}

One of the major determinants that characterize atherosclerotic inflammation is high production of cytokines and chemokines by cells of the immune system as well as endothelial and smooth muscle cells ${ }^{19)}$. High levels of cholesterol are associated with the development of atherosclerosis in animal models and in human studies, and it is therefore reasonable to expect the primary trigger of cytokine release could have been linked with the cholesterol accumulated in the aorta $^{27,28)}$. However, in this study we found that a CR had a robust effect on reducing cytokine expression in the aorta, and the effect was independent of dietary cholesterol. It is well known that different subfractions of LDL have different atherogenic potential ${ }^{29)}$. Smaller LDL particles are more atherogenic than the larger ones due to their longer plasma residence time and because their size allows them to penetrate the intima more readily where the atherosclerosis process begins ${ }^{30}$. Small dense LDL also has lower antioxidant content and is more susceptible to oxidation ${ }^{31)}$. Because of the increased concentration of small dense LDL in guinea pigs fed the high carbohydrate diets (Controls), there could have been greater penetration of these smaller LDL particles into the arterial wall where they became oxidized. There is sufficient evidence that oxidized LDL particles are potent inflammatory agents and posses a chemoattractant effect on monocytes and lymphocytes in addition to promoting the differentiation of monocytes into macrophages ${ }^{27,32)}$. This could be an 
explanation why the high carbohydrate groups had higher levels of cytokines and chemokines in the aorta. Another possible explanation of why high carbohydrate diets increased cytokines levels in the aorta could be linked to the reactivity of glucose with any proteins or lipoproteins. Glucose forms chemically irreversible glycated products with reactive amino groups of circulating lipoproteins or vessel wall proteins, generating advanced glycosylation end products (AGEs) ${ }^{33)}$. These glycasylation AGEs can induce modification of LDL particles and the vascular proteins and thus increase their atherogenicity or their normal functionality, respectively.

Finally, the third possible mechanism associated with the increase in inflammatory markers in the Control groups is the glycation of LDL particles. Glycosylation in the apoB of LDL occurs within the lysine residues, where the LDL receptor domain recognition is located $^{26)}$. Thus, glycated LDL are poorly recognized by the LDL receptor and removed from circulation by nonspecific receptor present on macrophages, lymphocytes and smooth muscle cells ${ }^{24)}$. In addition, the binding of AGE and LDL facilitates the oxidation of this lipoprotein, which stimulate the production of inflammatory cytokines $25,26,34,35)$. The different mechanisms aforementioned can account for the observation that Control diets contribute to the increase in aortic cytokine levels.

\section{Effects of Diet on Aorta's Mechanical Properties}

In the present study, we also indirectly evaluated the stiffness of the thoracic aorta and found that CR affected this parameter. When strain vs. stress-two important parameters in the determination of the stiffness of any material-was plotted, we observed that the arteries of the different dietary groups had different elastic properties. According to these curves we observed that the arteries from the CR-L and the Control-H groups were stiffer because they required more force per unit of area (stress) than the arteries of the Control-L and the CR-H groups to cause the same deformation (strain). This observation was also seen when a graph of modulus vs. stress was plotted (Fig. 4). The lines with the more pronounced slopes indicate stiffer arteries confirming that the Control-H and CR-L are stiffer since they have similar slopes.

The similar artery stiffness of the CR-L and Control-H groups might seem surprising in light of the previously observed finding that greater aorta stiffness is observed in advanced stages of artherosclerosis. However, studies conducted in diabetic rats and hypercolesterolemic-fed rabbits have shown that the relationship between atherosclerosis and arterial stiffness is not linear ${ }^{36,37)}$. Both studies mentioned that the normal artery is stiffer than the artery at the beginning of the atherosclerosis process. This is because at the onset of atherosclerosis, the lipid accumulation and the foam cell formation start causing arterial remodeling ${ }^{38)}$. As a result of the remodeling process, there is an increase in the activity of metalloproteinases and collagenases, which in turn degrades the collagen and the elastin of the arteries, increasing arterial compliance ${ }^{38,39)}$. However, in the event of prolonged diabetes or challenge with hypercholesterolemic diets, there is a higher increase in the rate of collagen synthesis (changing the collagen/elastin ratio) and an increase in the proliferation of smooth muscle cells, which in turn increases the stiffness of the artery ${ }^{17,40)}$. Therefore, the atherosclerosis process in relation to stiffness follows a parabolic curve as we indicated in Fig. 5. Hence, we may conjecture that even when the arterial stiffness of the CR-L and Control-H groups was similar, they are at different stages, with the CR-L group being at the normal stage or at the beginning of the atherosclerosis process, and the Control-H at the advanced stage. In the same way, because the Control-L and CR-H were similar in stiffness but softer than the other two groups, we may surmise that these two groups are located between the initial and the advanced stages of atherosclerosis.

Our data obtained from the other parameters of atherosclerosis supports our speculations. First, the Control-H group had higher levels of lipid accumulation in the aorta than the CR-L. Second, the Control-H group had higher concentrations of aortic inflammatory cytokines and higher sPLA 2 activity than the CR-L group and third the CR-L group had lower concentrations of small dense LDL. Thus, since the primary trigger of cytokine release is linked to the cholesterol accumulated in the aorta, and since higher ${ }_{s P L A}$ accelerates atherosclerosis, we may conclude that the Control-H was at the more advanced stage. In addition, recent studies have found that high plasma glucose increases the chances that glucose will react with collagen, so inducing the formation of intermolecular cross-links, which have a dramatic effect on the physical properties of the vascular system, and this could account for the markedly increase in arterial stiffness observed in diabetic subjects ${ }^{41,42}$. Although it had been suggested that AGEs formation was common only for diabetics, it is recognized that prolonged intake of simple sugars also increases the production of $\mathrm{AGEs}^{6,16)}$. This information supports the idea that the Control-H group was at a more advanced stage of atherosclerosis than the CR-L group. It also indicates that the combination of high carbohydrate intake and 
high cholesterol had the most adverse effects on the atherosclerotic process.

Even though stiffness was not different between Control-H and CR-L groups, the evaluation of the area under the curve indicates that the Control-H group presented worse mechanical properties characteristics of the aorta. This area is related with the amount of blood that the aorta can accommodate before the rupture. The greater the area, the greater the capability to accommodate the blood without rupture. The results showed that the amount of energy that the aorta from animals subjected to Control-H can absorb was significantly lower compared with CR-L animals. Therefore, the combination of high carbohydrate and high cholesterol diets favored the worsening of the biomechanical properties of the aorta.

In this study we have demonstrated that high concentrations of dietary cholesterol and high carbohydrate result in key modifications in the arteries involved in the initiation and progression of atherosclerosis. High cholesterol increases accumulation of lipids in the arteries plus increased activity of $\mathrm{sPLA}_{2}$ while high carbohydrate increases concentrations of aortic cytokines and chemokines plus affects the mechanical properties of the aorta that can be associated with different stages of the atherosclerotic process. Our results also show that CR attenuated the development of atherosclerosis even in the presence of high dietary cholesterol.

\section{References}

1) Bonow RO, Smaha LA, Smith SC Jr., Mensah GA, and Lenfant C: World Heart Day 2002: the international burden of cardiovascular disease: responding to the emerging global epidemic. Circulation, 2002; 106:1602-1605

2) Karsch KR: Atherosclerosis--where are we heading? Herz, 1992; 17:309-319

3) Dennis EA: Diversity of group types, regulation, and function of phospholipase A2. J Biol Chem, 1994; 269:1305713060

4) Farzaneh-Far A, Rudd J, and Weissberg PL: Inflammatory mechanisms. Br Med Bull, 2001; 59:55-68

5) Wang YX and Fitch RM: Vascular stiffness: measurements, mechanisms and implications. Curr Vasc Pharmacol, 2004; 2:379-384

6) Wuyts FL, Vanhuyse VJ, Langewouters GJ, Decraemer WF, Raman ER, and Buyle S: Elastic properties of human aortas in relation to age and atherosclerosis: a structural model. Phys Med Biol, 1995; 40:1577-1597

7) Zieman SJ, Melenovsky V, and Kass DA: Mechanisms, pathophysiology, and therapy of arterial stiffness. Arterioscler Thromb Vasc Biol, 2005; 25:932-943

8) Volek JS, Sharman MJ, and Forsythe CE: Modification of lipoproteins by very low-carbohydrate diets. J Nutr, 2005;
135:1339-1342

9) Flower RJ and Perretti M: Controlling inflammation: a fat chance? J Exp Med, 2005; 201:671-674

10) Fernandez ML and Volek JS: Guinea pigs: a suitable animal model to study lipoprotein metabolism, atherosclerosis and inflammation. Nutr Metab (Lond), 2006; 3:17

11) Zern TL, West KL, and Fernandez ML: Grape polyphenols decrease plasma triglycerides and cholesterol accumulation in the aorta of ovariectomized guinea pigs. J Nutr, 2003; 133:2268-2272

12) West KL, Zern TL, Butteiger DN, Keller BT, and Fernandez ML: SC-435, an ileal apical sodium co-dependent bile acid transporter (ASBT) inhibitor lowers plasma cholesterol and reduces atherosclerosis in guinea pigs. Atherosclerosis, 2003; 171:201-210

13) Lin EC, Fernandez ML, and McNamara DJ: Dietary fat type and cholesterol quantity interact to affect cholesterol metabolism in guinea pigs. J Nutr, 1992; 122:2019-2029

14) Torres-Gonzalez M, Volek JS, Sharman M, Contois JH, and Fernandez ML: Dietary carbohydrate and cholesterol influence the number of particles and distributions of lipoprotein subfractions in guinea pigs. J Nutr Biochem, 2006; 17:773-779

15) Reynolds LJ, Hughes LL, and Dennis EA: Analysis of human synovial fluid phospholipase A2 on short chain phosphatidylcholine-mixed micelles: development of a spectrophotometric assay suitable for a microtiterplate reader. Anal Biochem, 1992; 204:190-197

16) Nosaka T, Tanaka H, Watanabe I, Sato M, and Matsuda M: Influence of regular exercise on age-related changes in arterial elasticity: mechanistic insights from wall compositions in rat aorta. Can J Appl Physiol, 2003; 28:204-212

17) Hayashi K, Washizu T, Tsushima N, Kiraly RJ, and Nose Y: Mechanical properties of aortas and pulmonary arteries of calves implanted with cardiac prostheses. J Biomech, $1981 ; 14: 173-182$

18) Wood RJ, Volek JS, Liu Y, Shachter NS, Contois JH, and Fernandez ML: Carbohydrate restriction alters lipoprotein metabolism by modifying VLDL, LDL, and HDL subfraction distribution and size in overweight men. J Nutr, 2006; 136:384-389

19) Leitinger N, Watson AD, Hama SY, Ivandic B, Qiao JH, Huber J, Faull KF, Grass DS, Navab M, Fogelman AM, de Beer FC, Lusis AJ, and Berliner JA: Role of group II secretory phospholipase A2 in atherosclerosis: 2. Potential involvement of biologically active oxidized phospholipids. Arterioscler Thromb Vasc Biol, 1999; 19:1291-1298

20) Krauss RM: Dense low density lipoproteins and coronary artery disease. Am J Cardiol, 1995; 75:53B-57B

21) Millar JS and Packard CJ: Heterogeneity of apolipoprotein B-100-containing lipoproteins: what we have learnt from kinetic studies. Curr Opin Lipidol, 1998; 9:197-202

22) Caslake MJ, Packard CJ, Suckling KE, Holmes SD, Chamberlain P, and Macphee CH: Lipoprotein-associated phospholipase $\mathrm{A}(2)$, platelet-activating factor acetylhydrolase: a potential new risk factor for coronary artery disease. Atherosclerosis, 2000; 150:413-419

23) Piñón P and Kaski JC: [Inflammation, atherosclerosis and cardiovascular disease risk: PAPP-A, Lp-PLA2 and cystatin C. New insights or redundant information?]. Rev 
Esp Cardiol, 2006; 59:247-258

24) Bowie A, Owens D, Collins P, Johnson A, and Tomkin GH: Glycosylated low density lipoprotein is more sensitive to oxidation: implications for the diabetic patient? Atherosclerosis, 1993; 102:63-67

25) Bucala R, Makita Z, Vega G, Grundy S, Koschinsky T, Cerami A, and Vlassara H: Modification of low density lipoprotein by advanced glycation end products contributes to the dyslipidemia of diabetes and renal insufficiency. Proc Natl Acad Sci U S A, 1994; 91:9441-9445

26) Bucala R, Makita Z, Koschinsky T, Cerami A, and Vlassara $\mathrm{H}$ : Lipid advanced glycosylation: pathway for lipid oxidation in vivo. Proc Natl Acad Sci U S A, 1993; 90:64346438

27) Witztum JL and Steinberg D: Role of oxidized low density lipoprotein in atherogenesis. J Clin Invest, 1991; 88:1785-1792

28) Nilsson J: Regulating protective immunity in atherosclerosis. Circ Res, 2005; 96:395-397

29) Austin MA, Breslow JL, Hennekens CH, Buring JE, Willett WC, and Krauss RM: Low-density lipoprotein subclass patterns and risk of myocardial infarction. JAMA, 1988; 260:1917-1921

30) Lamarche B, Lemieux I, and Després JP: The small, dense LDL phenotype and the risk of coronary heart disease: epidemiology, patho-physiology and therapeutic aspects. Diabetes Metab, 1999; 25:199-211

31) Tribble DL, Rizzo M, Chait A, Lewis DM, Blanche PJ, and Krauss RM: Enhanced oxidative susceptibility and reduced antioxidant content of metabolic precursors of small, dense low-density lipoproteins. Am J Med, 2001; 110:103-110

32) Netea MG, Kullberg BJ, Demacker PN, Jacobs LE, VerverJansen TJ, Hijmans A, van Tits LH, Hoenderop JG, Willems PH, Van der Meer JW, and Stalenhoef AF: Native LDL potentiate TNF alpha and IL-8 production by human mononuclear cells. J Lipid Res, 2002; 43:1065-1071

33) Aronson D and Rayfield EJ: How hyperglycemia promotes atherosclerosis: molecular mechanisms. Cardiovasc
Diabetol, 2002; 1:1

34) Bucala R, Mitchell R, Arnold K, Innerarity T, Vlassara H, and Cerami A: Identification of the major site of apolipoprotein B modification by advanced glycosylation end products blocking uptake by the low density lipoprotein receptor. J Biol Chem, 1995; 270:10828-10832

35) Brownlee M, Cerami A, and Vlassara H: Advanced glycosylation end products in tissue and the biochemical basis of diabetic complications. N Engl J Med, 1988; 318:13151321

36) Zhao J, Lu X, Zhuang F, and Gregersen H: Biomechanical and morphometric properties of the arterial wall referenced to the zero-stress state in experimental diabetes. Biorheology, 2000; 37:385-400

37) Katsuda S, Machida N, Hasegawa M, Miyashita H, Kusanagi $\mathrm{M}$, Tsubone $\mathrm{H}$, and Hazama $\mathrm{A}$ : Change in the static rheological properties of the aorta in Kurosawa and Kusanagi-hypercholesterolemic (KHC) rabbits with progress of atherosclerosis. Physiol Meas, 2004; 25:505-522

38) Cai WJ, Koltai S, Kocsis E, Scholz D, Kostin S, Luo X, Schaper W, and Schaper J: Remodeling of the adventitia during coronary arteriogenesis. Am J Physiol Heart Circ Physiol, 2003; 284:H31-H40

39) Keeling WB, Armstrong PA, Stone PA, Bandyk DF, and Shames ML: An overview of matrix metalloproteinases in the pathogenesis and treatment of abdominal aortic aneurysms. Vasc Endovascular Surg, 2005; 39:457-464

40) Shanahan CM and Weissberg PL: Smooth muscle cell phenotypes in atherosclerotic lesions. Curr Opin Lipidol, 1999; 10:507-513

41) Schram MT, Henry RM, van Dijk RA, Kostense PJ, Dekker JM, Nijpels G, Heine RJ, Bouter LM, Westerhof N, and Stehouwer CD: Increased central artery stiffness in impaired glucose metabolism and type 2 diabetes: the Hoorn Study. Hypertension, 2004; 43:176-181

42) Sims TJ, Rasmussen LM, Oxlund H, and Bailey AJ: The role of glycation cross-links in diabetic vascular stiffening. Diabetologia, 1996; 39:946-951 